\title{
INTERACTION BETWEEN AN INFINITE STRINGER AND TWO IDENTICAL PRESTRESSED STRIPS: CONTACT PROBLEM
}

\author{
V. B. Rudnitskii and N. N. Dikhtyaruk
}

\begin{abstract}
The plane contact problem of the transfer of a horizontal concentrated load from an infinite stringer to two identical prestressed strips clamped at one edge is solved using the linearized theory of elasticity. The solution is found in general form for the theory of large initial deformations and different theories of small initial deformations for an arbitrary elastic potential. The problem for the normal and tangential contact stresses is reduced to a system of integro-differential equations derived using the Fourier transform. The contact stresses are represented by Fourier integrals. It is shown that the initial stresses in the strips affect strongly the distribution of contact stresses: the contact stresses substantially decrease under compression and increase under tension, whereas the displacements increase under compression and decrease under tension. The effect of the initial stresses is stronger in highly elastic materials than in stiff material
\end{abstract}

Keywords: plane contact problem, strip-stringer-strip system, linearized theory of elasticity, initial (residual) stress, initial displacement

Introduction. One of the factors having a strong effect on the reliability and life of engineering facilities, mechanical structures, and machine parts is initial (residual) stresses. In this connection, the study of the contact interaction of prestressed elastic bodies is still of relevance $[12,13]$. There are many publications dealing with static problems of classical elasticity and closely related to the subject of the present paper. Many of them are closely related to Melan's classical study [14]. The uniqueness and importance of [14] is in addressing fundamental problems for a half-plane and a strip. Similar problems for a prestressed half-plane were analyzed by Guz [1].

The contact interaction of between a prestressed half-plane and pads is studied in $[2,6,8]$. The effect of the prestresses in an elastic strip on its contact interaction with elastic pads was analyzed in [3-5]. In what follows, we will study the contact interaction between an infinite stringer and two (rather than one) prestressed strips.

1. Problem Statement. Basic Equations. Using the linearized theory of elasticity $[1,15,16]$, we will formulate and solve the problem of load transfer from an infinite stringer to two identical prestressed strips. The strips are elastic, infinite, made of identical compressible (or incompressible) materials with an arbitrary elastic potential, subject to equal initial or residual stresses, have thickness $t=$ const, clamped at the edges, joined by an infinite elastic stringer with elastic modulus $E_{1}$ and Poisson's ratio $v_{1}$, and subject to a horizontal force $\alpha \rightarrow \infty$ applied at the middle of the stringer $\left(\delta\left(y_{1}\right)\right.$ is the known Dirac delta). We will use the coordinates of the initial stress-strain state (Fig. 1).

We will determined the distribution of the normal and tangential contact stresses along the interface line between the stringer and the prestressed strips. According to $[2,15,16]$, we assume that well-known principles $1-4$ hold, which underlie the theory of the contact interaction of prestressed bodies. We also assume that the load and tangential contact stresses stretch or compress the stringer as a rod (that is in a uniaxial stress state $[8,14]$ ) and that the vertical elastic displacements are constant along the horizontal axis, which is due to the small thickness of the stinger. Denoting the intensities of the normal and tangential

Khmelnytskyi National University, 11 Institutskaya St., Khmelnytskyi, Ukraine 29016, e-mail: massacran2@ukr.net. Translated from Prikladnaya Mekhanika, Vol. 53, No. 2, pp. 41-48, March-April, 2017. Original article submitted December 27, 2015. 


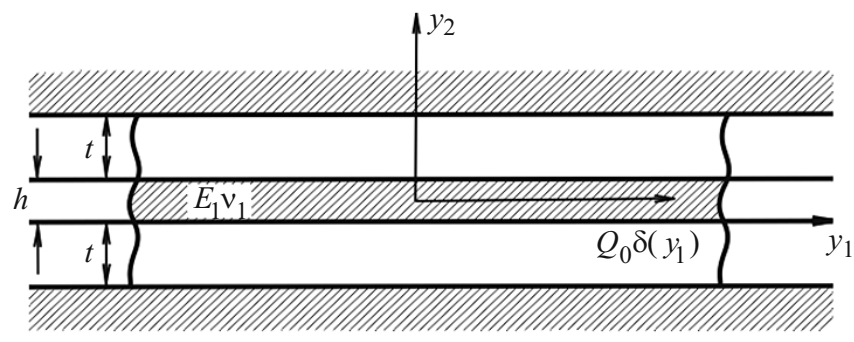

Fig. 1

contact stresses by $p\left(y_{1}\right)$ and $q\left(y_{1}\right)$ and the vertical and horizontal displacements of the stringer by $v^{(1)}\left(y_{1}\right)^{(1)}$ and $u^{(1)}\left(y_{1}\right)$, we can write the following equalities:

$$
\begin{gathered}
\frac{\partial u^{(1)}\left(y_{1}\right)}{\partial y_{1}}=\frac{1}{E_{1} h} \int_{-\infty}^{y_{1}}\left[2 q(t)-Q_{0} \delta(t)\right] d t \quad\left(-\infty<y_{1}<\infty\right), \\
\frac{\partial v^{(1)}\left(y_{1}\right)}{\partial y_{1}}=0 \quad \forall y_{1} \in\left(-\infty<y_{1}<\infty\right) .
\end{gathered}
$$

The boundary conditions on the contact line are perfect bonding:

$$
\frac{\partial v^{(1)}\left(y_{1}\right)}{\partial y_{1}}=\frac{\partial u_{2}^{(2)}\left(y_{1}\right)}{\partial y_{1}}, \quad \frac{\partial u^{(1)}\left(y_{1}\right)}{\partial y_{1}}=\frac{\partial u_{1}^{(2)}\left(y_{1}\right)}{\partial y_{1}} \quad\left(-\infty<y_{1}<\infty\right),
$$

where $u^{(1)}\left(y_{1}\right), v^{(1)}\left(y_{1}\right)$ are the components of the displacement vector in the stringer; $u_{1}^{(2)}\left(y_{1}\right), u_{2}^{(2)}\left(y_{1}\right)$ are the components of the displacement vector in the strips. With conditions (1.1), (1.2), (1.3), the vertical and horizontal displacements of the nonclamped edge $y_{1}=0$ are expressed as follows [3-5]:

$$
\begin{aligned}
& u_{1}\left(y_{1}\right)=\int_{-\infty}^{\infty} h_{11}\left(\left|y_{1}-t\right|\right) p(t) d t+\int_{-\infty}^{\infty} h_{12}\left(y_{1}-t\right) q(t) d t, \\
& u_{2}\left(y_{1}\right)=\int_{-\infty}^{\infty} h_{21}\left(y_{1}-t\right) p(t) d t+\int_{-\infty}^{\infty} h_{22}\left(\left|y_{1}-t\right|\right) q(t) d t .
\end{aligned}
$$

2. Reducing the Problem to a System of Singular Integro-Differential Equations. Using (1.1)-(1.4) for the unknown contact stresses $p(t)$ and $q(t)$ and omitting the transformations, we obtain the following system of integro-differential equations:

$$
\begin{gathered}
\frac{d}{d y_{1}}\left[\int_{-\infty}^{\infty} h_{11}\left(\left|y_{1}-t\right|\right) p(t) d t+\int_{-\infty}^{\infty} h_{12}\left(y_{1}-t\right) q(t) d t\right]=0, \\
\frac{d}{d y_{1}}\left[\int_{-\infty}^{\infty} h_{21}\left(y_{1}-t\right) p(t) d t+\int_{-\infty}^{\infty} h_{22}\left(\left|y_{1}-t\right|\right) q(t) d t\right]=\int_{-\infty}^{y_{1}}\left[2 q(t)-Q_{0} \delta(t)\right] d t,
\end{gathered}
$$

where $h_{i j}(i, j=1,2)$ are the influence functions for a prestressed strip that are expressed [3-5], depending on the type of load, as follows: 